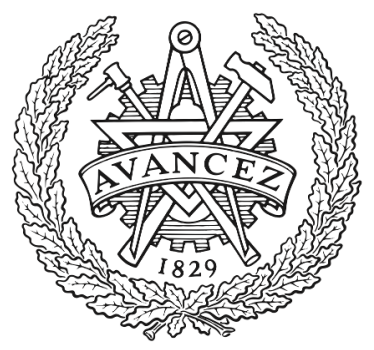

CHALMERS

UNIVERSITY OF TECHNOLOGY

\title{
Overview of the Origins Space telescope: Science drivers to observatory requirements
}

Downloaded from: https://research.chalmers.se, 2023-04-26 13:07 UTC

Citation for the original published paper (version of record):

Meixner, M., Armus, L., Battersby, C. et al (2018). Overview of the Origins Space telescope: Science drivers to observatory requirements. Proceedings of SPIE - The International Society for Optical Engineering, 10698. http://dx.doi.org/10.1117/12.2312255

N.B. When citing this work, cite the original published paper. 


\section{Overview of the Origins Space telescope: science drivers to observatory requirements}

\section{Margaret Meixner, Lee Armus, Cara Battersby, James Bauer, Edwin Bergin, et al.}

Margaret Meixner, Lee Armus, Cara Battersby, James Bauer, Edwin Bergin, Asantha Cooray, Jonathan J. Fortney, Tiffany Kataria, David T. Leisawitz, Stefanie N. Milam, Klaus Pontoppidan, Alexandra Pope, Karin Sandstrom, Johannes G. Staguhn, Kevin B. Stevenson, Kate Y. Su, Charles Matt Bradford, Dominic Benford, Denis Burgarella, Sean Carey, Ruth C. Carter, Elvire De Beck, Michael J. Dipirro, Kimberly Ennico-Smith, Maryvonne Gerin, Frank P. Helmich, Lisa Kaltenegger, Eric. E. Mamajek, Gary Melnick, Samuel Harvey Moseley, Desika Narayanan, Susan G. Neff, Deborah Padgett, Thomas L. Roellig, Itsuki Sakon, Douglas Scott, Kartik Sheth, Joaquin Vieira, Martina Wiedner, Edward Wright, Jonas Zmuidzinas, "Overview of the Origins Space telescope: science drivers to observatory requirements," Proc. SPIE 10698, Space Telescopes and Instrumentation 2018: Optical, Infrared, and Millimeter Wave, 106980N (24 July 2018); doi: 10.1117/12.2312255 


\section{Overview of the Origins Space Telescope: Science Drivers to Observatory Requirements}

Margaret Meixner ${ }^{\mathrm{a}, \mathrm{b}, \mathrm{c}}$, Lee Armus ${ }^{\mathrm{d}}$, Cara Battersby ${ }^{\mathrm{e}}$, James Bauer ${ }^{\mathrm{f}}$, Edwin Bergin ${ }^{\mathrm{g}}$, Asantha Cooray ${ }^{\mathrm{h}}$, Jonathan J. Fortney ${ }^{\mathrm{i}}$, Tiffany Kataria ${ }^{\mathrm{j}}$, David T. Leisawitz ${ }^{\mathrm{c}}$, Stefanie N. Milam ${ }^{\mathrm{c}}$, Klaus Pontoppidan ${ }^{\mathrm{a}}$, Alexandra Pope ${ }^{\mathrm{k}}$, Karin Sandstrom ${ }^{1}$, Johannes G. Staguhn ${ }^{\mathrm{b}, \mathrm{c}}$, Kevin B. Stevenson ${ }^{\mathrm{a}}$, Kate Y. Su ${ }^{\mathrm{m}}$, Charles Matt Bradford ${ }^{\mathrm{j}}$, Dominic Benford ${ }^{\mathrm{n}}$, Denis Burgarella ${ }^{\mathrm{o}}$, Sean Carey ${ }^{\mathrm{d}}$, Ruth C. Carter ${ }^{\mathrm{c}}$, Elvire De Beck ${ }^{\mathrm{p}}$, Michael J. DiPirro ${ }^{\mathrm{c}}$, Kimberly Ennico-Smith ${ }^{\mathrm{q}}$, Maryvonne Gerin ${ }^{\mathrm{r}}$, Frank P. Helmich ${ }^{\mathrm{s}}$, Lisa Kaltenegger, Eric E. Mamajek ${ }^{\mathrm{j}}$, Gary J. Melnick ${ }^{\mathrm{u}}$, Samuel Harvey Moseley ${ }^{\mathrm{c}}$, Desika Narayanan ${ }^{\mathrm{v}}$, Susan G. Neff ${ }^{\mathrm{c}}$, Deborah Padgett $\mathrm{t}^{\mathrm{j}}$, Thomas L. Roellig ${ }^{\mathrm{q}}$, Itsuki Sakon ${ }^{\mathrm{w}}$, Douglas Scott ${ }^{\mathrm{x}}$, Kartik Sheth ${ }^{\mathrm{n}}$, Joaquin Vieira ${ }^{\mathrm{y}}$, Martina Wiedner ${ }^{\mathrm{r}}$, Edward Wright ${ }^{\mathrm{z}}$, Jonas Zmuidzinas ${ }^{\mathrm{A}}$

${ }^{a}$ Space Telescope Science Institute, Baltimore, MD, USA

${ }^{\mathrm{b}}$ Johns Hopkins University, Baltimore, MD, USA

${ }^{c}$ NASA Goddard Space Flight Center, 8800 Greenbelt Rd., Greenbelt, MD 20771 USA

${ }^{\mathrm{d}}$ Caltech/Infrared Processing and Analysis Center, Pasadena, CA, USA

${ }^{\mathrm{e}}$ Department of Physics, University of Connecticut, Storrs, CT, USA

${ }^{\mathrm{f}}$ Astronomy Department, University of Maryland, College Park, MD, USA

${ }^{g}$ Department of Astronomy, University of Michigan, Ann Arbor, MI, USA

${ }^{\mathrm{h}}$ Department of Physics and Astronomy, University of California, Irvine, Irvine, CA, USA

${ }^{i}$ Astronomy and Astrophysics Dept., University of California, Santa Cruz, Santa Cruz, CA, USA

${ }^{j}$ Caltech/Jet Propulsion Laboratory, Pasadena, CA, USA

${ }^{\mathrm{k}}$ Department of Astronomy, University of Massachusetts, Amherst, MA, USA

${ }^{1}$ CASS, University of California, San Diego, San Diego, CA, USA

${ }^{m}$ Dept. of Astronomy and Steward Observatory, The University of Arizona, Tucson, AZ, USA

${ }^{\mathrm{n}}$ Astrophysics Division, Science Mission Directorate, NASA Headquarters, Washington, DC, USA

${ }^{\circ}$ Laboratoire d'Astrophysique de Marseille, Marseille, France

${ }^{\mathrm{p}}$ Dept. of Space, Earth and Environment, Chalmers Institute of Technology, Gothenburg, Sweden

${ }^{q}$ NASA Ames Research Center, Mountain View, CA, USA

${ }^{\mathrm{r}}$ Sorbonne Université, Observatoire de Paris, CNRS, LERMA, F-75014, Paris, France

${ }^{\mathrm{s}}$ Netherlands Institute for Space Research (SRON), Groningen, The Netherlands

${ }^{t}$ Carl Sagan Institute at Cornell, Ithaca, NY, USA

${ }^{u}$ Harvard-Smithsonian Center for Astrophysics, Cambridge, MA, USA

${ }^{v}$ Department of Astronomy, University of Florida, Gainesville, FL, USA

${ }^{\mathrm{w}}$ School of Science, The University of Tokyo, 7-3-1 Hongo, Bunkyo-ku, Tokyo, Japan

${ }^{x}$ Dept. of Physics and Astronomy, The University of British Columbia, Vancouver, BC, Canada

${ }^{y}$ Department of Astronomy, University of Illinois, Urbana-Champaign, Urbana, IL, USA

${ }^{\mathrm{z}}$ Division of Astronomy and Astrophysics, UCLA, Los Angeles, CA, USA

${ }^{A}$ Division of Physics, Mathematics and Astronomy, Caltech, Pasadena, CA, USA

\section{ABSTRACT}

The Origins Space Telescope (OST) mission concept study is the subject of one of the four science and technology definition studies supported by NASA Headquarters to prepare for the 2020 Astronomy and Astrophysics Decadal

Space Telescopes and Instrumentation 2018: Optical, Infrared, and Millimeter Wave, edited by Makenzie Lystrup, Howard A. MacEwen, Giovanni G. Fazio, Proc. of SPIE Vol. 10698, 106980N (C) 2018 SPIE · CCC code: $0277-786 X / 18 / \$ 18 \cdot$ doi: $10.1117 / 12.2312255$ 
Survey. OST will survey the most distant galaxies to discern the rise of metals and dust and to unveil the co-evolution of galaxy and blackhole formation, study the Milky Way to follow the path of water from the interstellar medium to habitable worlds in planetary systems, and measure biosignatures from exoplanets. This paper describes the science drivers and how they drove key requirements for OST Mission Concept 2, which will operate between $\sim 5$ and $\sim 600$ microns with a JWST sized telescope. Mission Concept 2 for the OST study optimizes the engineering for the key science cases into a powerful and more economical observatory compared to Mission Concept 1.

\section{INTRODUCTION}

The Origins Space Telescope (OST) is a large mission concept study for the NASA 2020 Astronomy and Astrophysics Decadal Study. In our 2016 SPIE paper $^{1}$, we described our science and technology ideas for the FarInfrared (Far-IR) Surveyor, the mission study name given by the 2013 NASA Astrophysics Roadmap, Enduring Quests, Daring Visions ${ }^{2}$ and used in the first community report. ${ }^{3}$ Our first science exercise for the Science and Technology Definition Team (STDT) involved a community driven science proposals to determine the most scientifically compelling questions for the 2030s. The NASA selected STDT members voted on the proposals and the top 14 were used to deicide on architecture, large aperture telescope or interferometer. The resulting large aperture telescope became OST Mission Concept 1's $9.1 \mathrm{~m}$ telescope, with 5 powerful instruments covering the 5 to $660 \mu \mathrm{m}$ and capable of addressing all the science questions posed by the community. One of the science questions, searching for biosignatures from habitable zone exoplanets transiting $\mathrm{M}$ dwarfs, was ranked in the top 3 and extended the wavelength range to include the 5-28 $\mu \mathrm{m}$ window. The Far-Infrared Surveyor no longer described the mission. Through an internal team renaming competition, we selected Origins Space Telescope (OST) to capture and focus the attention on the science themes that extend from the first stars to life. The OST Mission Concept 1 is described in the interim report that will become public this summer $2018^{4}$. At this 2018 SPIE, we are presenting for the first time Mission Concept 2.

The goal of our OST mission concept study is a large astronomy mission concept capable of exciting science that is technologically executable in the 2030s. Both the science definition and the technical implementation are important to the study. In this paper, we provide a brief overview of the OST Mission Concept 2 in section 2. In sections 3, 4 and 5 of the paper we describe the science drivers for some key observatory requirements that are defining Mission Concept 2. We end in section 6 with a summary and plan for completion of the final report due to NASA HQ in 2019.

\section{OST MISSION CONCEPT 2 OVERVIEW}

Mission Concept 2 for the OST study (Figure 1) optimizes the engineering for the key science cases into a powerful and more economical observatory compared to Mission Concept $1 .^{5}$ The telescope has a JWST size collecting area, $\sim 25 \mathrm{~m}^{2}$, and segmented with keystone shaped segments resulting in a circular aperture. ${ }^{5,6,7}$ The telescope has a diffraction limit of $30 \mu \mathrm{m}$ and covers the wavelength range of $\sim 5$ to $\sim 620 \mu \mathrm{m}$. The telescope and instruments are kept cold at $\sim 4 \mathrm{~K}$ and the specific instrument detectors are cooled to lower temperatures. ${ }^{8,9}$ The observatory can move fast, up to 100 arcseconds per second, and the detectors can keep up with the fast motion resulting in impressive surveying capabilities. The observatory would be launched in one of three large rockets under development, NASA SLS 8.4 m, SpaceX Big Falcon Rocket and Blue Origins New Glenn. The space in the large rockets allows the observatory to be launched with minimal deployment (sunshields, and the usual deployments like solar panels). 


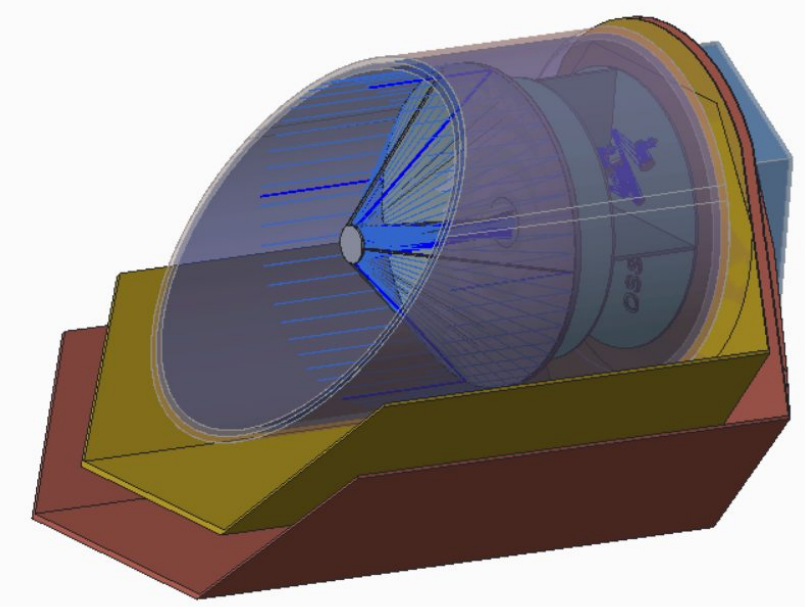

Figure 1: The OST Mission Concept 2, conceptual design. The light path shown in blue rays.

Mission Concept 2 has at most four optimized instruments. Astronomers view point for instruments and the observatory is the instrument focal plane (Figure 2) that is used to plan observations. The instruments are discussed in detail in other contributions of this conference and we only briefly cover their capabilities here. The Far-infrared Imager and Polarimeter (FIP) ${ }^{10}$ does broad band imaging at 50, 100, 250 and $500 \mu \mathrm{m}$, two wavebands in parallel at a time, over large angular areas. FIP also does polarimetric imaging in the bands. The Mid-infrared Imager, Spectrometer and Coronagraph (MISC) ${ }^{11}$, which operates between 5 and $28 \mu \mathrm{m}$, has an ultra-stable spectrometer channel built to do exoplanet transits with high precision ${ }^{12}$ (smaller MISC field of view). The MISC imager (larger field of view) serves both as a science imager and a guider for the observatory. The OST Survey Spectrometer $(\mathrm{OSS})^{13}$ can survey the sky over its whole wavelength range of 25 and $590 \mu \mathrm{m}$ with low resolution spectroscopy with R $(\lambda / \Delta \lambda)$ of $\sim 300$. OSS has two higher spectral resolution modes: a fourier transform spectrometer (FTS) with $\mathrm{R} \sim 43,000$ at $112 \mu \mathrm{m}$ and an etalon with $\mathrm{R} \sim 325,000$ at $112 \mu \mathrm{m}$. The Heterodyne Receiver for OST (HERO) ${ }^{14}$ uses an array of 9 coherent detectors over the wavelength range of 111 to $617 \mu \mathrm{m}$ to achieve the highest spectral resolutions of $\mathrm{R} \sim 10^{6}$ to $10^{7}$ for measurements of simultaneous spectral lines.

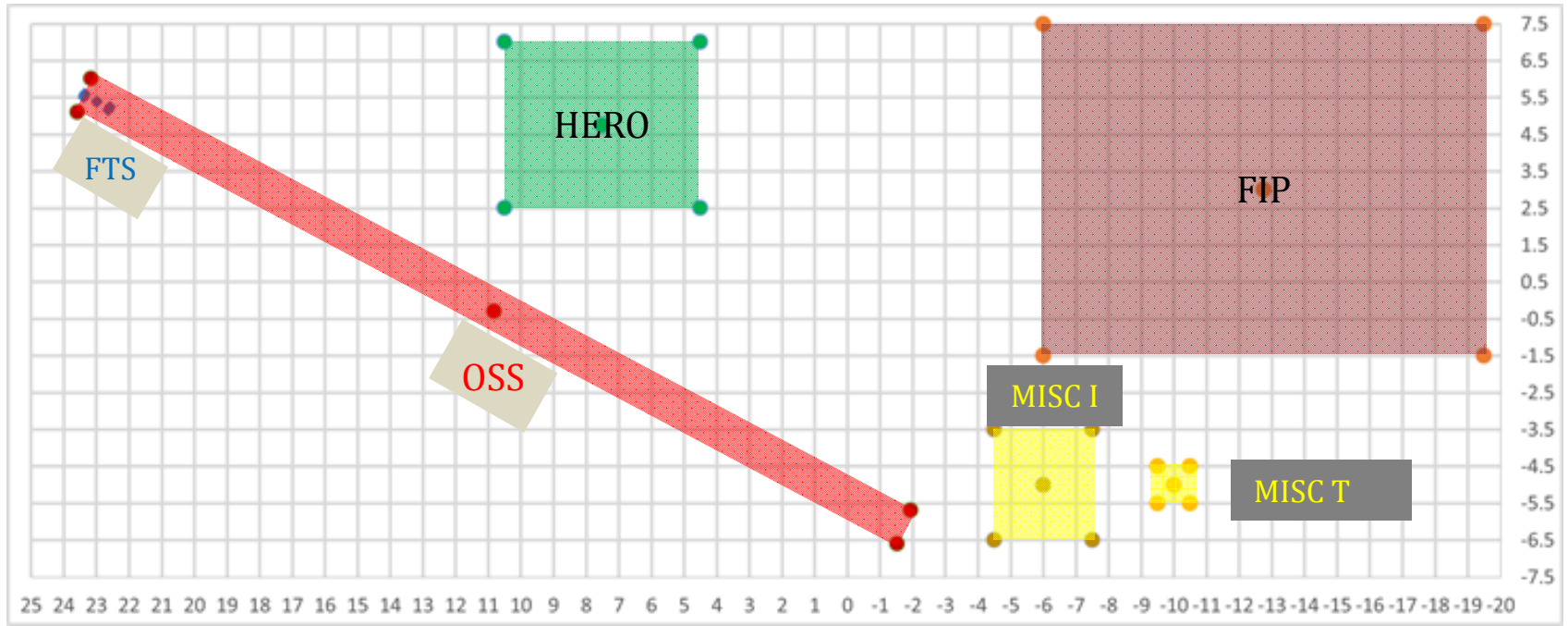

Figure 2: The astronomers view of the Origins Space Telescope Mission Concept 2, the field of view focal plane. In clockwise order, the instruments are the Far-infrared Imager and Polarimeter (FIP, brown box), the Mid-Infrared, Spectrometer and Camera (MISC, Imager is large yellow box and small yellow box is the transit spectrometer), the OST Survey Spectrometer (OSS, long pink slit) and the Heterodyne Receiver for OST (HERO, green box). 


\section{ARE WE ALONE?}

One of the most compelling scientific questions for humanity is "Are We Alone?" The OST mission study will address the more specific question "How common are life-bearing planets orbiting M dwarf stars?" Using the MISC ultra-stable transit spectrometer on OST, astronomers will search for bio-signatures in the atmospheres of nearby terrestrial exoplanets through transmission and emission spectroscopy (Figure 3). The mid-infrared spectral domain has two biosignatures, ozone $\left(\mathrm{O}_{3}\right)$ and methane $\left(\mathrm{CH}_{4}\right)$, that if found in an exoplanet atmosphere confirms life as we know it. ${ }^{15}$ Astrochemists have shown and observed that ozone or methane alone can be produced through non-biological means but to have them occur together requires life in the processes. The mid-infrared spectral domain also has two bioindicators, carbon dioxide $\left(\mathrm{CO}_{2}\right)$ and water $\left(\mathrm{H}_{2} \mathrm{O}\right)$, that are important supporters of life as we know it. Together this midinfrared spectral range is rich for this work.

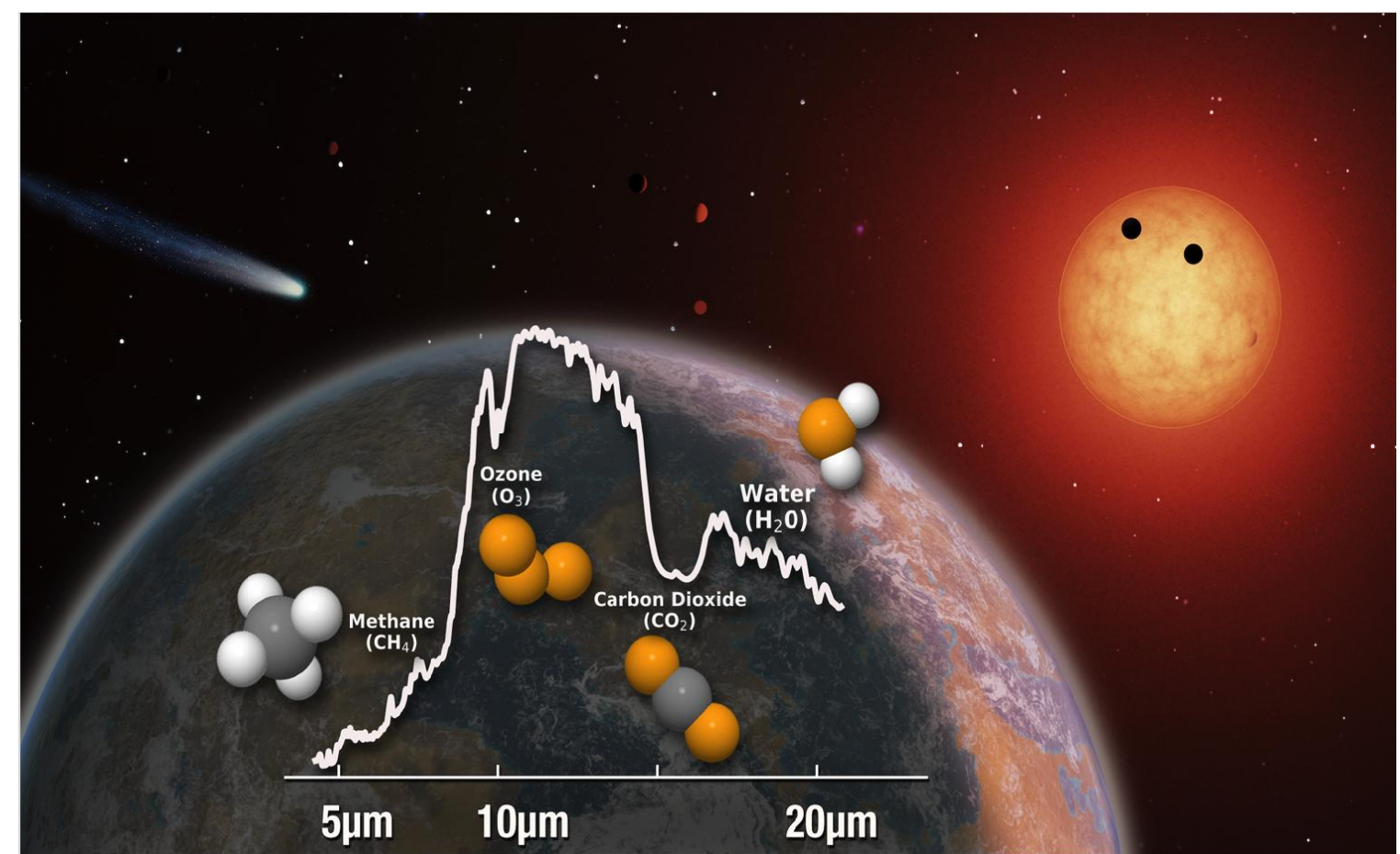

Figure 3: With its ultra-stable transit spectrometer, OST will characterize bio-signatures (ozone and methane) and bioindicators (carbon dioxide and water) in the atmospheres of nearby exoplanets orbiting M stars to determine if we are alone in the Universe.

We anticipate that the James Webb Space Telescope (JWST) will be making some initial forays into searching for biosignatures in nearby exoplanets and that many additional habitable zone planet candidates beyond TRAPPIST-1 will be identified before the 2030s. The exoplanet community will have honed their skills in transit spectroscopy even more and prepare them for OST's MISC spectrometer's exciting research opportunity. Moreover transit spectroscopy offers a rich amount of information about the system. The masses and radii are precisely determined for both star and planet down to $5 \%$ if also measured by the Gaia astrometric mission. Therefore bulk densities will be known for planetary candidates and predominantly rocky planets in the habitable zone can be selected with great confidence. M dwarf stars are a rich target pool because $75 \%$ of stars within 15 pc are $M$ dwarfs and mid-to-late $M$ dwarfs provide the best signal when searching for life. Exoplanet transits around the smaller $\mathrm{M}$ dwarf stars have larger transit depths, closer habitable zones (5-100 days) and increased transit probability in the habitable zone. 
The JWST Mid-InfraRed Instrument (MIRI) spectrometers (LRS and MRS) will produce many, highly significant break throughs for astronomers. However, exoplanet transit science was just starting when the requirements and development of MIRI were set, and the MIRI spectrometers were not required to achieve nor designed for the highly demanding biosignature transit science we propose. This important science goal drives the following requirements for OST:

1. Telescope size: $25 \mathrm{~m}^{2}$ collecting area (JWST size), $5.9 \mathrm{~m}$ diameter

2. Wavelength coverage: $4-25 \mu \mathrm{m}$

3. High stability mid-IR spectroscopy: 5 ppm, R 300 (MISC transit spectrometer)

The collecting area is set both by simulations of anticipated data and by anticipating that JWST collecting area for transit work will set an expectation for astronomers for this work. The wavelength coverage is set by the wavelengths of the key bio-signatures and bio-indicators. OST will be colder increasing the sensitivity at the longer wavelength range over JWST and the increased in stability of the instrument, requirement \#3 above, is the real gain over prior instruments.

The exoplanet team on OST have simulated TRAPPIST-1e-like transmission and emission spectra for both JWST MIRI/LRS and OST MISC transit channel (Figure $4 \mathrm{a}$ and $\mathrm{b}$ ). During primary transit, the planet is detected passing in front of its host star and a transmission spectrum is obtained. During conjunction or secondary eclipse, when a planet passes behind its host star, OST will measure the planet's thermal emission, i.e. emission spectrum. The emission from a planet in the habitable zone, as well as the planet-to-star flux ratio, peaks in the mid-infrared. The assumptions for the simulation include 100 visits and the $\mathrm{M}$ dwarf is reasonably bright with a $\mathrm{K}$ ( 2.12 micron) magnitude of 8 . Based on documentation of expected MIRI/LRS performance, the optimistic noise is 30 parts per million (ppm). For OST/MISC transit channel, we set a requirement of 5 ppm necessary to measure the bio-signatures.

The key technology development for the MISC transit channel is stable detectors with reasonable size format so that the spectra can be calibrated to $5 \mathrm{ppm}$, which is very demanding.

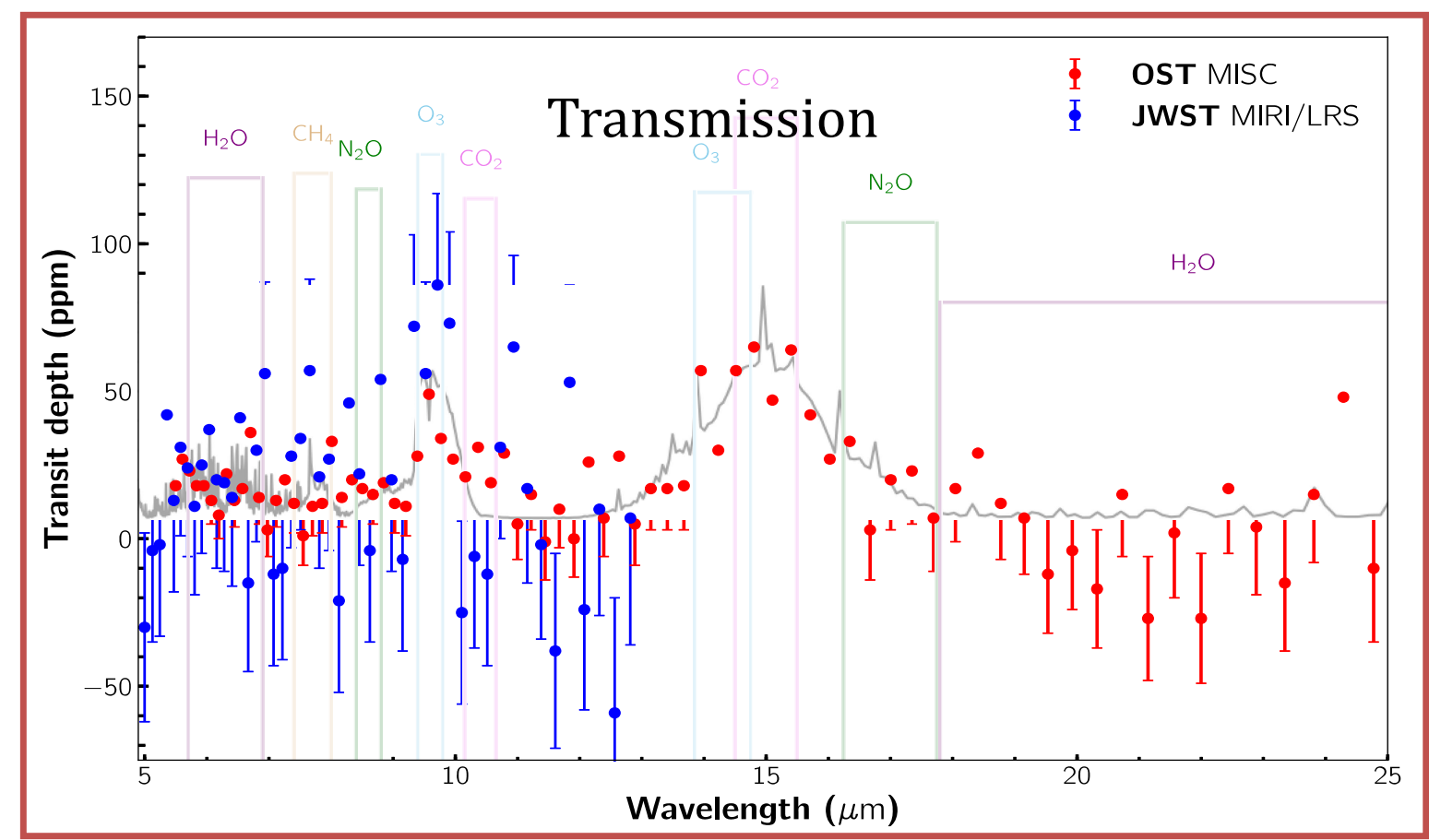

Figure 4a: The simulated TRAPPIST-1 e-like transmission spectrum assuming 100 visits. Comparing JWST MIRI/LRS with the OST MISC transit channel. 


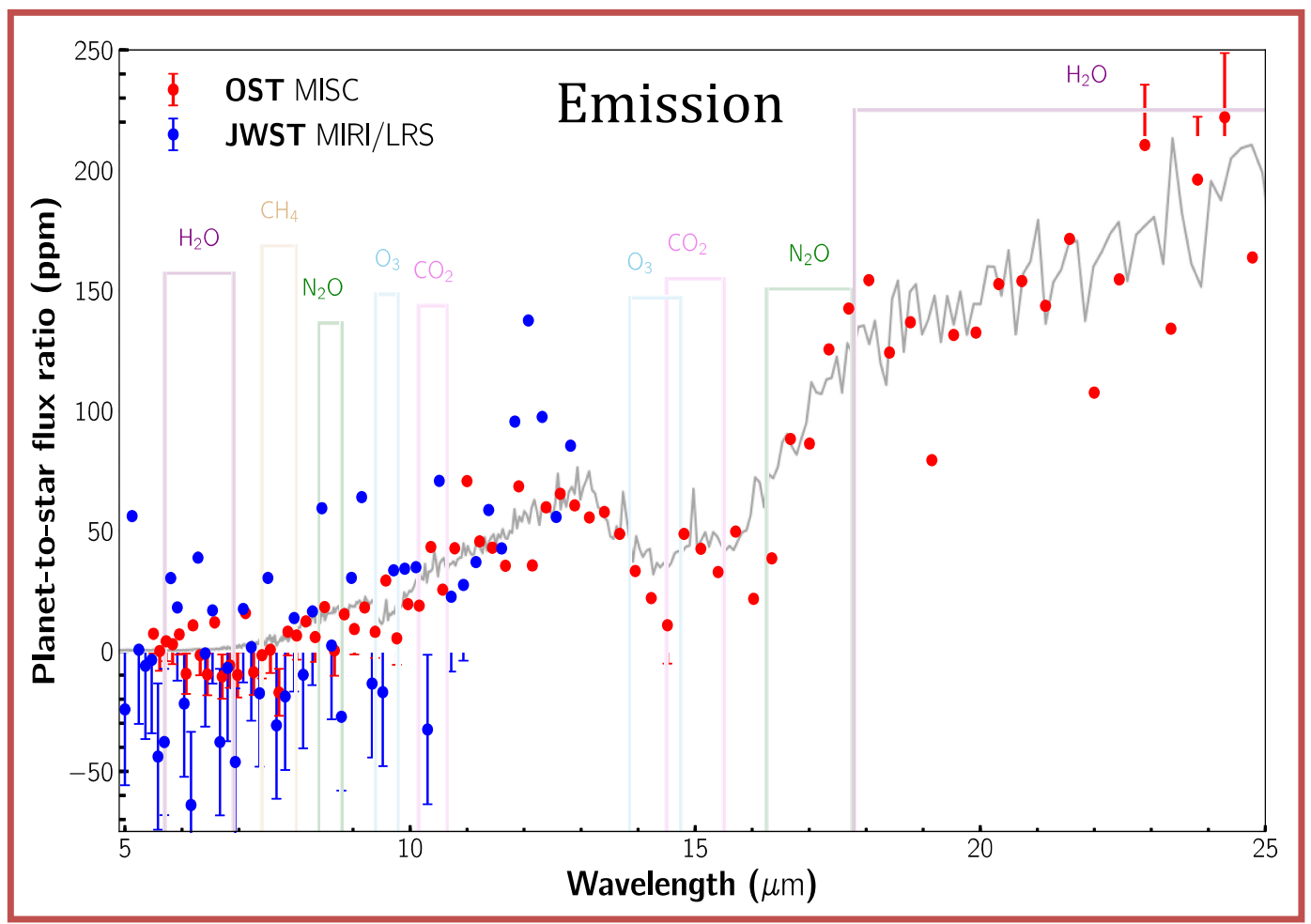

Figure 4b: The simulated TRAPPIST-1e-like emission spectrum assuming 100 visits. Comparing JWST MIRI/LRS with the OST MISC transit channel.

\section{HOW DID WE GET HERE?}

We know there is life in the Universe, because Earth is teaming with it. So an equally compelling question is, "How did we get here?" OST will unveil the whole story of the origins of habitable planetary systems by following the trail of water and gas from the cold interstellar medium, through the formation of the stars to the resulting proto-planetary disk forming planets. Prior work with observatories such as the Herschel Space Observatory ${ }^{16}$, reveal water and gas in a few proto-planetary disks. OST will enable large statistical samples of disks required to bring true understanding.

The OST will specifically address the question: "How do the conditions for habitability develop during the process of planet formation?" The spectral range of OSS covers the whole spectrum of water lines and the key gas mass tracer, $112 \mu \mathrm{m}$ HD line. (Figure 5). Astronomers will use the OSS to survey the water ( $179 \mu \mathrm{m} \mathrm{H}_{2} \mathrm{O}$ line) and measure the total gas mass (112 $\mu \mathrm{m}$ HD line) in 1000 planet-forming disks around stars of all masses and evolutionary stages out to the distance of Orion. Using high resolution spectroscopy available with OSS and HERO, astronomers will measure the distribution of water and gas mass in 200 disks selected from the survey.

Figure 6 shows an artistic model of a protoplanetary disk undergoing the planetary system formation process. The gas mass content of the disks can be measured with the HD $112 \mu \mathrm{m}$ line. The dust content of these protoplanetary disks has been extensively studied using the Spitzer Space Telescope ${ }^{17}$ and the warm dust will also be explored with JWST. However, the bulk of the disk mass is in the form of gas, and most of it is located in the cold outter disk. OST's long wavelength coverage is required to measure the bulk of the disk gas. The life supporting water of a disk is in the form of ice or gas and the OST spectrometers will be capable of measuring both. Using the highest spectral resolution of the OST spectrometers, OSS and HERO, we can use the technic of Doppler tomography to determine the location of the water vapor in the disk. The material in the disk will follow Keplerian rotation and hence the slower material will reside 
in the outer part of the disk compared with the faster moving gas in the inner disk. Thus, OST's OSS and HERO spectroscopy will define the total water content of the disk and its location.

Key technical requirements to support this case include:

1. Wavelength coverage $28-200 \mu \mathrm{m}$ for survey (OSS), 28-540 $\mu \mathrm{m}$ for followup (HERO)

2. Spectral line sensitivity sensitivity: $1.0 \times 10^{-21} \mathrm{~W} \mathrm{~m}^{-2}$ at 112 and $179 \mu \mathrm{m}$ (OSS)

3. Spectral resolving power: 43,000 for survey and 200,000 for followup (OSS, HERO)

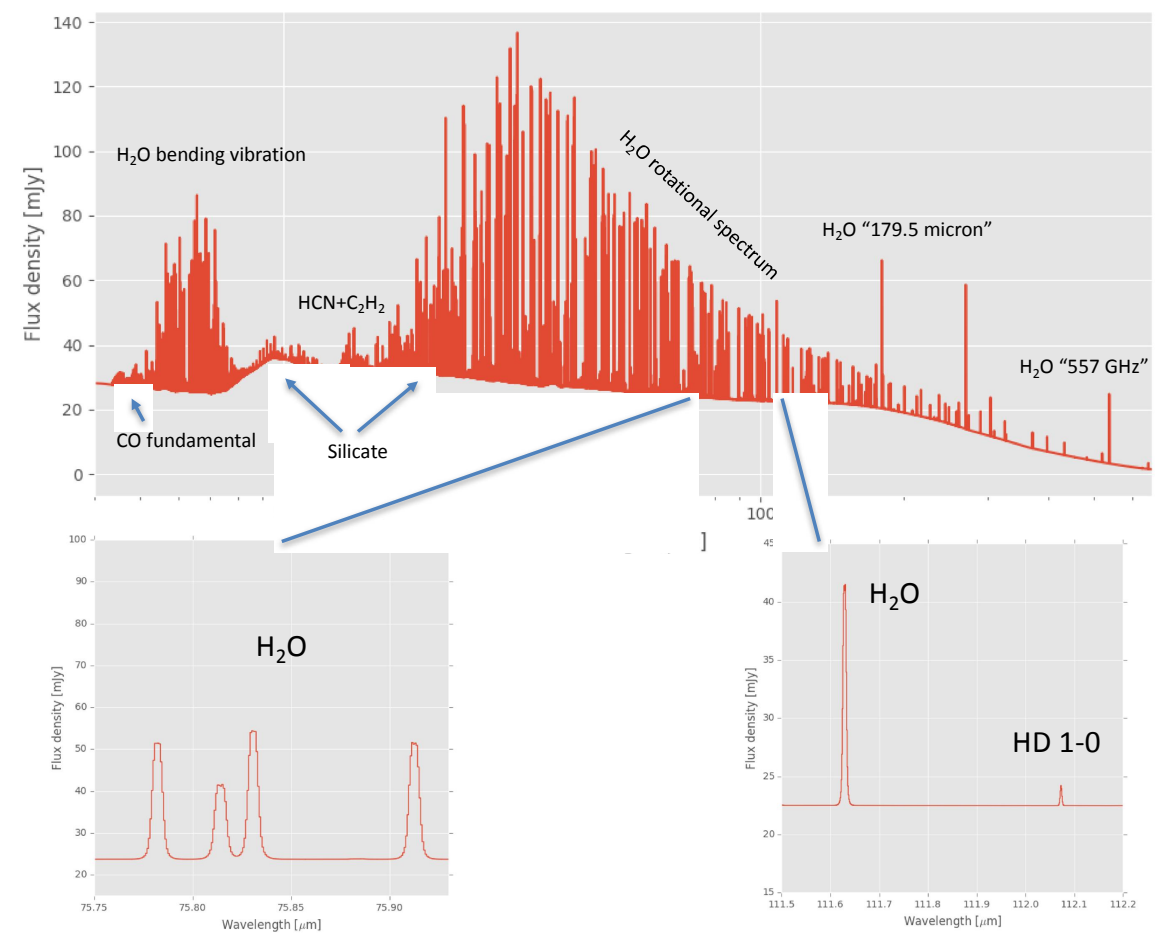

Figure 5: A model spectrum of a proto-planetary disk showing the water lines and other important gas lines (such as HD 112 $\mu \mathrm{m})$ that are accessed by the spectral coverage of the OSS instrument.

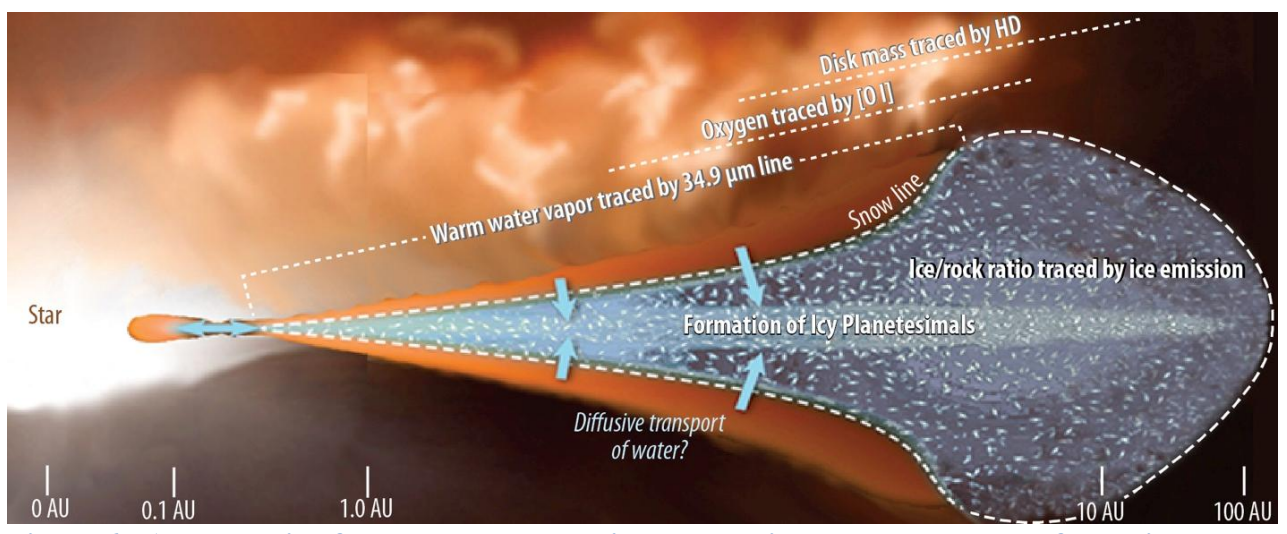

Figure 6: A schematic of a proto-planetary disk undergoing planetary system formation. Most of the water in the form of ice or vapor exists in the outside of the disk, where as the habitability zone is closer to $1 \mathrm{AU}$. How is the water transported? Noted in the diagram are the locations in the disk of the HD line which traces disk gas mass, OI to measure oxygen abundance, and one of the water lines. Only the long wavelengths of OST can access the outside disk. 
The line sensitivity to measure the faint lines in the proto-planetary disks requires the telescope to be chilled to $4 \mathrm{~K}$ and for significant improvements in far-infrared direct detector sensitivities (Figure 7). The cryogenic design of OST ${ }^{9}$ uses cryo-coolers to make the system cold. Thanks to the development invested in the crycooler for JWST/MIRI this technology is fairly advanced and in need of modest investment. Our technical tall pole for these measurements are the far-infrared direct detectors.

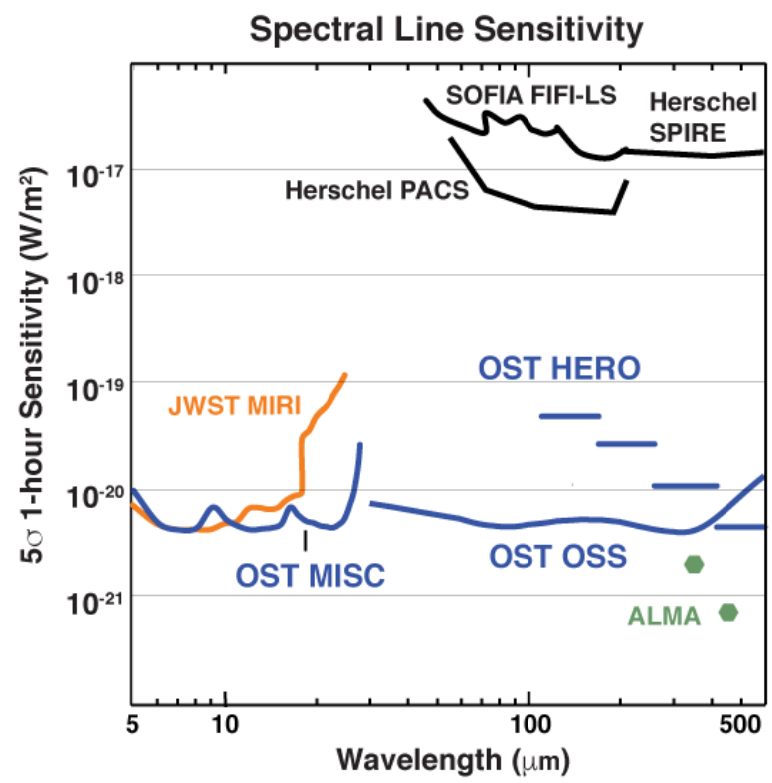

Figure 7: The spectral line sensitivity for the three OST spectrometers: MISC transit, OSS and HERO, in comparison to prior, current and near future facilities. The three orders of magnitude increase in sensitivity is created by making OST cold $(\sim 4 \mathrm{~K})$ and required improvements in the far-infrared detectors. MISC sensitivity improves over JWST MIRR at the long wavelengths, but the real gain for the transit spectrometer is the stability of the instrument.

\section{HOW DOES THE UNIVERSE WORK?}

The Universe is red-shifted and OST will be able to extend astronomers' JWST investigations of galaxy evolution to higher red-shifts and to measuring the galaxies' inter-stellar medium, which is the fuel of star formation (Figure 8). The high-z galaxy investigations fall under the broad question, "How does the Universe work?" OST will specifically address the question, "How do galaxies form stars, grow their central supermassive black holes, and make heavy elements over cosmic time?" OST will simultaneously measure the spectral energy distribution of $>10^{6}$ galaxies out to z $=10$, and the metallicity, dust and black hole accretion rate in samples of $10^{5}$ galaxies $\left(>2.5 \times 10^{11} \mathrm{~L}_{\odot}\right)$ out to $\mathrm{z}=5$, unencumbered by dust obscuration. The observation strategies will employ wide field imaging surveys with FIP, area $2000 \mathrm{deg}^{2}$, and wide field spectral surveys with OSS, area $\sim 10 \mathrm{deg}^{2}$.

Galaxy evolution involves the co-evolution of star formation and black hole evolution (observed as active galactic nuclei, AGN). In addition the rise of metals and dust in galaxies and the universe comes from the life cycle of stars in galaxies. The peak epoch of star formation at redshift of 2 and the epoch of reionization at redshift of 6 mark key points in the evolution of galaxies. The OST wavelength coverage fills in a critical gap in wavelength between JWST and 
ALMA that is needed to form a complete understanding of galaxy evolution (Figure 8). The OST OSS spectrometer is specifically designed to capitalize on this wavelength range allowing spectral surveys of deep fields.

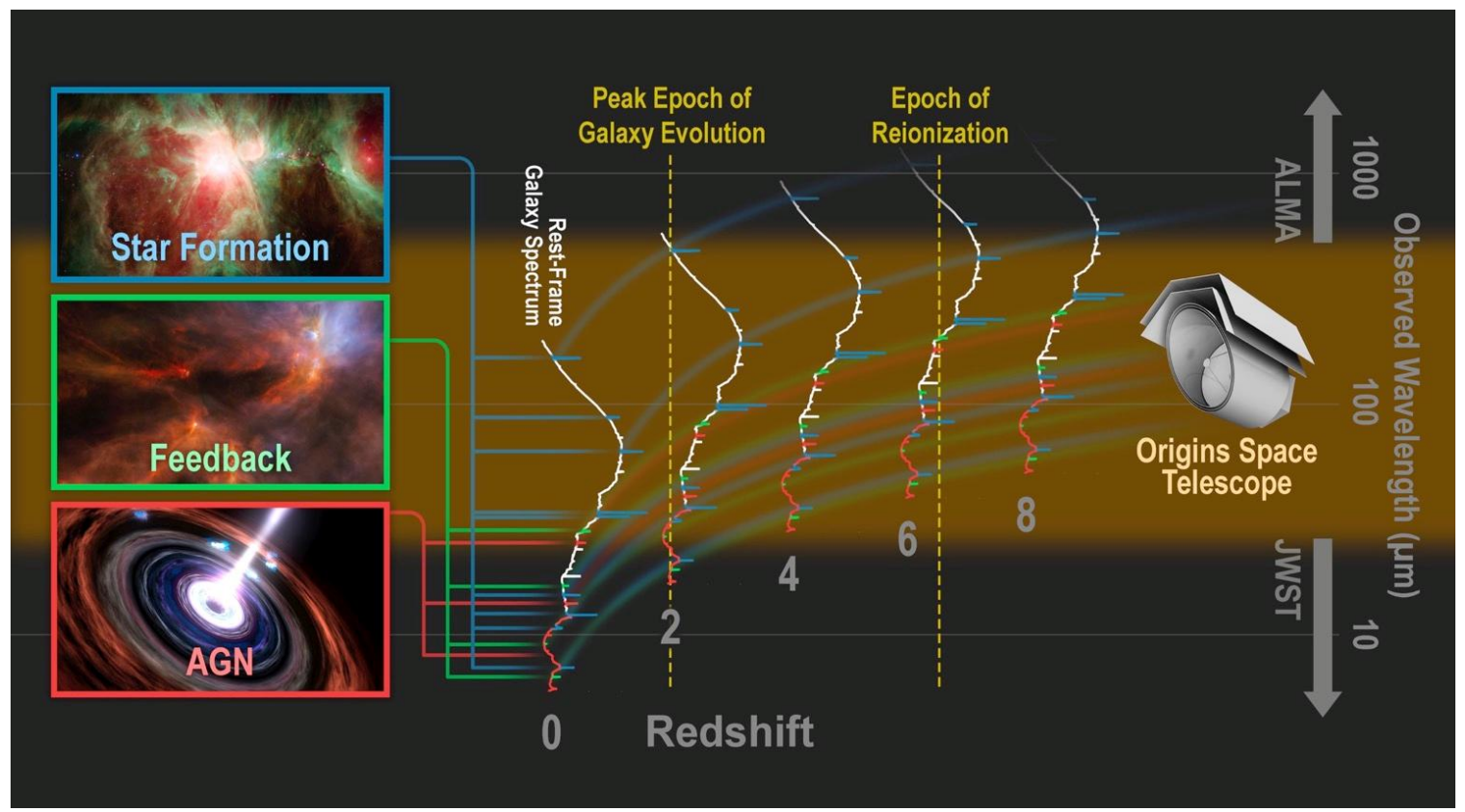

Figure 8: A schematic plot of observed wavelength vs. redshift for galaxy spectral energy distributions show that OST fills in a critical wavelength gap between JWST and ALMA. The white line galaxy spectral energy distribution is one of a nearby galaxy with both active star formation and an active galactic nuclei (AGN). The colored spectral lines trace the activity of star formation (blue), feedback from star formation or AGN (green) and AGN (red).

The OST is designed for fast mapping of the sky in part because of the detectors functioning and in part because the confusion limit of this highly sensitive telescope is reached in seconds with FIP. The significantly improved image quality over prior missions (Figure 9) will enable better identification of counterparts. Large area surveys of the sky are becoming the mainstay of galaxy evolution and large scale structure of the universe research with LSST, Euclid and WFIRST coming online. OST with FIP will complement these studies by providing the longer wavelength measurements that measure the interstellar medium of the galaxy thereby creating large samples of galaxies for significant statistical study. Moreover, the large area surveys of $2000 \mathrm{deg}^{2}$ will reveal the rarer, most luminous galaxies for study.

These extragalactic surveys levy several requirements on the instruments and observatory:

1. Wavelength coverage 5-28 $\mu \mathrm{m}$ (MISC), 25-590 $\mu \mathrm{m}$ (OSS), 50, 100, 250 and $500 \mu \mathrm{m}$ (FIP)

2. Spectral line sensitivity : $5.0 \times 10^{-21} \mathrm{~W} \mathrm{~m}^{-2} @ 150 \mu \mathrm{m}(\mathrm{OSS})$

3. Spectral resolving power: 300 for spectral survey and 20,000 for followup (OSS)

4. Fast mapping, survey speed: 100 arcseconds per second

Several of these are repeats from the proto-planetary disks science. However, the fast mapping speed is new and enables large area sky surveys which differentiates it from JWST. The key technology development for this science is farinfrared direct detectors for sensitivity. 

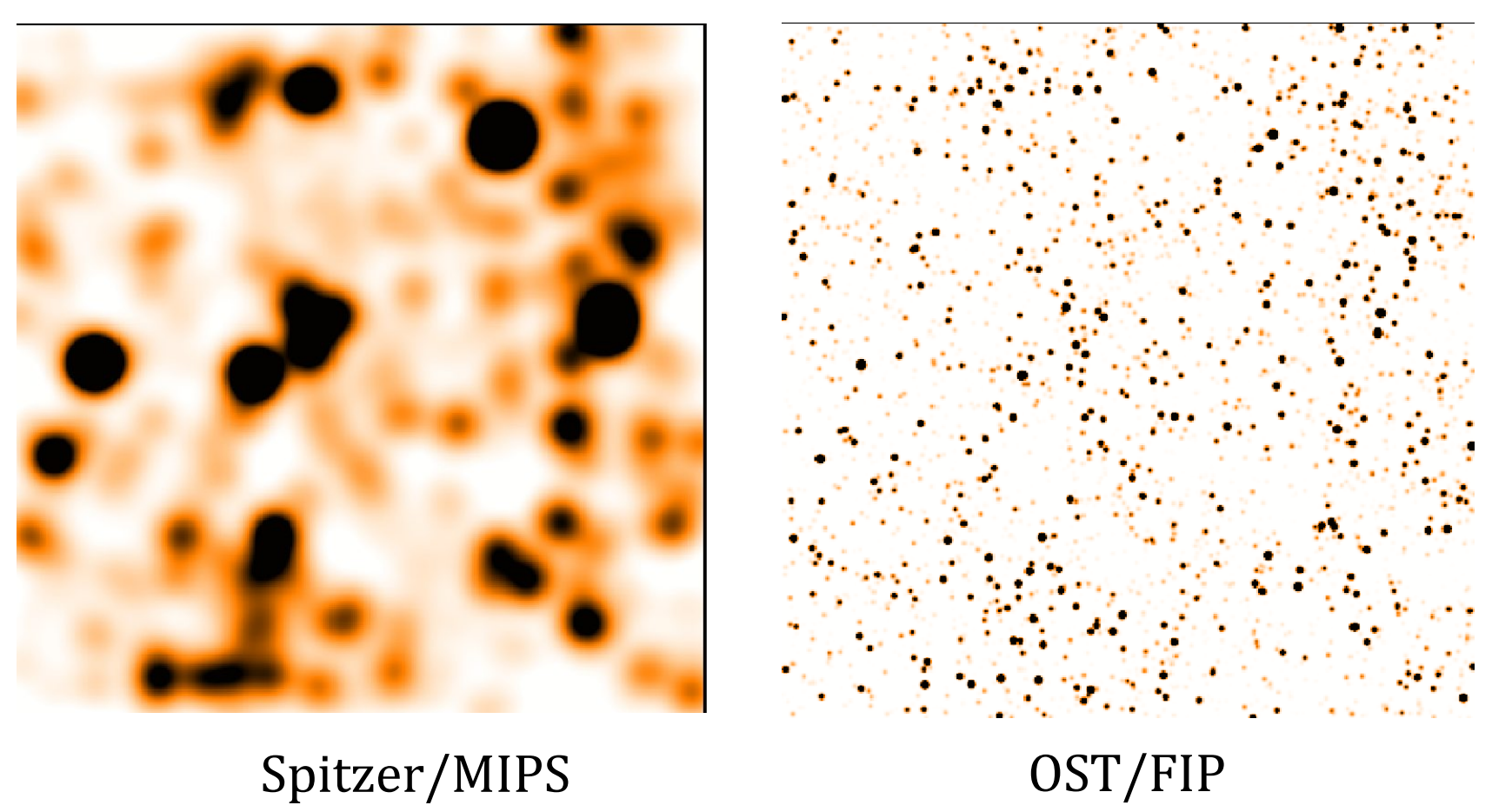

Figure 9: Comparison of a simulated deep galaxy field as imaged with Spitzer/MIIPS and OST/FIP. The field of view is 5.25 ' $x$ 5.25'.

\section{FUTURE}

The OST mission concept 2 will be complete its engineering study this summer 2018. The OST decadal study will submit its final report for NASA HQ in winter 2019. These final reports will be delivered to the National Academy in summer 2019 by NASA HQ. Our OST study is an open process and the community is welcomed to join us. If this paper makes you curious about us, then please join us. To learn more about us, visit our outreach website at origins.ipac.caltech.edu or if you want to listen in on our telecons, visit the project website

asd.gsfc.nasa.gov/firs/.

\section{REFERENCES}

[1] Meixner, M., Cooray, A., Carter, R., DiPirro, M., Flores, A., Leisawitz, D., Armus, L., Barttersby, C., Bergin, E., Bradford, C.M., Ennico, K., Melnick, G.J., Milam, S., Narayanan, D., Pontoppidan, K., Pope, A., Roellig, T., Sandstrom, K., Su, K.Y.L., Vieira, J., Wright, E., Zmuidzinas, J., Alato, S., Carey, S., Gerin, M., Helmich, F., Menten, K., Scott, D., Sakon, I., Vavrek, R., 2016, SPIE, 9904, 0, “The Far-Infrared Surveyor Mission study: paper I, the genesis" 
[2] NASA 2013 Science Roadmap Team (Kouveliotou, C., Chair), "Enduring Quests, Daring Visions: NASA Astrophysics in the Next Three Decades," http://science.nasa.gov/media/medialibrary/2013/12/20/secureAstrophysics_Roadmap 2013.pdf

[3] Armus,L., Bauer, J., Benford, D., et al. "From Early Galaxies to Habitable Planets: The Science Case and Concept for a Far-Infrared Surveyor,” June 2015, http://conference.ipac.caltech.edu/firsurveyor/

[4] Origins Space Telescope: From first stars to life, 2018 Interim report, by the Science and Technology Definition Team (STDT) of the Origins Space Telescope study, led by M. Meixner and A. Cooray. Published online asd.gsfc.nasa.gov/firs/, origins.ipac.caltech.edu and NASA HQ Decadal Studies site.

[5] Leisawitz, D. T. et al. the OST STDT and Study team 2018, SPIE, "The Origins Space Telescope: mission concept overview"

[6] Lightsey, P., et al. 2018, SPIE, "Stray Light overview for the Origins Space Telescope"

[7] Knight, S. et al. 2018, SPIE, “OST wavefront error budget flowdown"

[8] Arenberg, J. W., Pohner, J., Harpole, G., et al. 2018, SPIE, "Thermal considerations and architecture for Origins Space Telescope"

[9] Dipirro, M. J., et al. 2018, SPIE, “The Origins Space Telescope cryogenic-thermal architecture”

[10] Staguhn, J. G. et al. 2018, SPIE, “Origins Space Telescope: the far infrared imager and polarimeter FIP”

[11] Sakon, I., et al. 2018, SPIE, "The mid-infrared imager/spectrometer/coronagraph instrument (MISC) for the Origins Space Telescope"

[12] Matsuo, T., et al. 2018, SPIE, "A high stable spectrophotometric capability for the Origins Space Telescope (OST) Mid-Infrared Spectrometer and Camera,"

[13] Bradford, C.M., Cameron, B., Moore, B.D. et al. 2018, SPIE, “The Origins Survey Spectrometer (OSS): a far-IR discovery machine for the Origins Space Telescope"

[14] Wiedner, M. et al. 2018, SPIE, "HERO: heterodyne receiver for the Origins Space Telescope"

[15] Kaltenegger, L. 2017, ARA\&A 55, 433, "How to Characterize Habitable Worlds and Signs of Life"

[16] Pilbratt, G. L., Riedinger, J.R., Passvogel, T. et al. 2010, A\&A, 518, L1, "Herschel Space Observatory. An ESA facility for far-infrared and submillimeter astronomy"

[17] Werner, M.W., Roellig, T.L., Low, F.J., et al. 2004, ApJS, 154, 309, “The Spitzer Space Telescope Mission” 